\title{
Normal modes and stability of phase separated trapped Bose-Einstein condensates
}

\author{
Anatoly A. Svidzinsky and Siu-Tat Chui \\ Bartol Research Institute, University of Delaware, Newark, DE 19716
}

(October 11, 2018)

\begin{abstract}
We study the normal modes and the stability of two component condensates in a phase separated regime. In such a regime the system can undergo a quantum phase transition upon the change of interaction strength between bosons of the same species or the variation of the trap frequencies. In this transition, the distribution of the two components changes from a symmetric to an asymmetric shape. We discuss the nature of the phase transition, the role of the interface tension and the phase diagram. The symmetric to asymmetric transition is the simplest quantum phase transition that one can imagine. We found new branches of normal modes which are specific for trapped multicomponent condensates and are analogous to the waves at the interface between two layers of immiscible fluids under gravity. At the point of the phase transition the frequencies of those modes go to zero and become imaginary which causes an instability. The interface tension shifts the normal mode frequencies and changes the stability region of the system.
\end{abstract}

\section{INTRODUCTION}

There is much recent interest in Bose-Einstein condensation (BEC) in trapped gases as well as quantum phase transitions (QPT). Examples of the QPT include the Wigner electron solid melting transition, the Mott-Hubbard metal-insulator transition, and different magnetic transitions. In physical phenomena involving BECs quantum mechanics play a crucial role. In this paper we propose a new kind of quantum phase transition in phase-separated mixtures of BECs. In this transition, the distribution of the two components changes from a symmetric to an asymmetric shape. To explore the transition, we first investigate the stability of the symmetric phase by studying its normal modes. We find interface modes that become soft. These unstable modes can be caused by changing the trapping potential and are analogous to gravitational waves at the interface between two immiscible fluids. When the lowest frequency becomes zero, the instability sets in which determines the stability limit of the symmetric phase. We determine the actual phase boundary by comparing the energy between the symmetric phase and the asymmetric phase and find that the actual phase boundary and the instability boundary is not the same. This suggests that the transition is first order. The system may be a good laboratory to study issues of quantum metastability and tunnelling. The symmetric to asymmetric transition is the simplest quantum phase transition that one can imagine. Careful study of this problem should provide us new insight into this burgeoning field of discovery.

Systems of multicomponent condensates were first realized by the JILA group in a magnetic trap in ${ }^{87} \mathrm{Rb}$ [1,2] and subsequently in an optical trap in ${ }^{23} \mathrm{Na}$ (spinor condensate) [3, 4 ]. Experimentally at low fields, the spin exchange process can occur in an optically trapped condensate, leading to spin domains [4] with metastable behavior [5.6]. Binary condensates in two hyperfine levels of ${ }^{87} \mathrm{Rb}$ have been studied [1],7], most notably realizing a system of interpenetrating Bose fluids [2], measurements of phase dispersion [8], and a vortex state in a dilute-gas BEC [9]. Theoretical treatment of such systems began in the context of superfluid helium mixtures $\sqrt{10}$ and spin-polarized hydrogen [11], and now has been extended to BEC in trapped gases [12, 13, 14, 15, 16, 17, 18, 19, 20, 21].

The equilibrium density distributions of segregated mixtures in the absence of gravity have been studied numerically for different system parameters. Two types of configurations have been discussed: a symmetric [17,20, 14 configuration, for which one component is inside the other one, and an asymmetric one in which the two components occupy the left and the right hand side of a sphere 20,14. Several theoretical papers have described collective excitations of multicomponent condensates. Busch et al. 222] studied collective excitations in the limit of weak interactions based on a trial function approach. Collective excitations of binary mixtures in the Thomas-Fermi (TF) limit have been investigated by Graham and Walls [23. Esry and Greene 24 based on the Hartree-Fock and random-phase approximation have numerically calculated the low-lying excitations of double BECs in a time-averaged orbiting potential trap in which gravity separates the centers of the two components. Gordon and Savage [25] have studied excitation spectrum as a function of number of particles. Mazets [26] has performed an analysis of wave dispersion on a boundary between two weakly segregated untrapped BECs.

Under a change of the interaction strength the two component BECs can undergo different types of macroscopic quantum phase transitions. For example, an increase in interspecies interactions $a_{12}$ results in a transition from a 
binary mixture to a phase separated state. Such phase separated condensates are analogous to a system of immiscible fluids with positive surface tension. $\mathrm{Pu}$ and Bigelow 27 numerically studied the frequency of collective excitations as a function of interspecies interactions $a_{12}$ and found appearance of imaginary normal mode frequencies at the phase separation transition point. Such imaginary modes describe the nature of system's instability. The trapping potential is not crucial for this type of phase transition and similar imaginary modes also exist in the homogeneous (untrapped) BECs 15, 19,23.

However, in the phase separated regime the trapping potential changes the system's symmetry and results in a new type of quantum phase transition. Such transition occurs in trapped condensates upon the change of relative intraspecies interaction strength $a_{11} / a_{22}$. When this ratio differs significantly from one the less repulsive component is in the middle of the trap and the more repulsive component comprises outer shell. One can make the initial configuration unstable by manipulating the ratio $a_{11} / a_{22}$ close to one, e.g., by means of Feschbach resonances or by changing the ratio of the trapping frequencies. In this paper we study the collective excitations of the trapped phase separated condensates as a function of $a_{11} / a_{22}$ and show that normal modes with imaginary frequencies appear when this ratio approaches one. Effect of the interface tension results in a shift of the transition point of the symmetricasymmetric transition in the two component BEC. In general, the asymmetric phase possesses a lower interface energy. On the other hand, since the degree of self-repulsion may differ between the two species, the less self-repulsive component will prefer to remain where the density is higher, while the other component moves to the low density regions outside. This favors the symmetric phase. Depending on the system parameters, one of these two energetic considerations will win out. These system parameters can be adjusted by changing the trapping frequencies, the relative particle numbers of the two species, and the interaction between the particles with Feschbach resonances. We first address the stability of the symmetric phase.

\section{BASIC FORMALISM}

Let us consider the two-component BEC in a spherically symmetric trap. The dynamics of the system is described by time dependent Gross-Pitaevskii equations

$$
\begin{aligned}
& i \hbar \frac{\partial \Psi_{1}}{\partial t}=-\frac{\hbar^{2}}{2 m} \Delta \Psi_{1}+V_{\mathrm{tr}} \Psi_{1}+\frac{4 \pi \hbar^{2}}{m}\left(a_{11}\left|\Psi_{1}\right|^{2}+a_{12}\left|\Psi_{2}\right|^{2}\right) \Psi_{1}, \\
& i \hbar \frac{\partial \Psi_{2}}{\partial t}=-\frac{\hbar^{2}}{2 m} \Delta \Psi_{2}+V_{\mathrm{tr}} \Psi_{2}+\frac{4 \pi \hbar^{2}}{m}\left(a_{22}\left|\Psi_{2}\right|^{2}+a_{12}\left|\Psi_{1}\right|^{2}\right) \Psi_{2},
\end{aligned}
$$

where $\Psi_{1,2}$ are the condensate wave functions, $V_{\mathrm{tr}}=m \omega_{0}^{2} r^{2} / 2$ is the trapping potential, $\omega_{0}$ is the trapping frequency, $r$ is the radial spherical coordinate, $a_{i j}>0$ are $s$-wave scattering lengths. Here we suppose that trapping frequencies and particle masses are the same for both species. We discuss the general case in the last section.

We shall assume in this paper that the condition

$$
a_{12}^{2}-a_{11} a_{22}>0
$$

is satisfied and, therefore, the condensates are phase-segregated. The first demonstration of a condensate binary mixture by Myatt et. al. [1] produced overlapping condensates of the $\mid F=1, m_{f}=-1>$ and $\mid F=2, m_{f}=2>$ spin states of ${ }^{87} \mathrm{Rb}$. These states, however, possess different magnetic moments and, hence, the condensates experience different potentials in a magnetic trap which results in unequal displacement from the trap center by gravity. Later JILA experiments were performed on mixtures of $\mid 1,-1>$ and $\mid 2,1>$ states [2]. These two states have essentially identical magnetic moments, and feel identical confining potentials. In ${ }^{87} \mathrm{Rb}$, the scattering lengths for $\mid 1$, $-1>$ and $\mid 2,1>$ states are known to be in the proportion $a_{11}: a_{12}: a_{22}:: 1.03: 1: 0.97$, with the average of the three

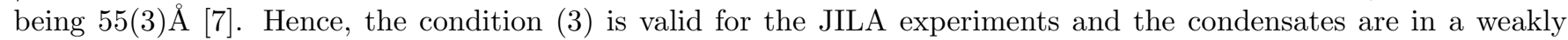
segregated phase [18]. One should mention that for earlier experiments with the $\mid 1,-1>$ and $\mid 2,2>$ rubidium states the scattering lengths are $a_{11}: a_{12}: a_{22}:: 1.007: 1: 1.01$ [28]. For such states the condition (3) is not fulfilled and the mixture exhibits behavior of a miscible system.

We suppose the condensates can be well described by the TF approximation. In this regime, the phase-segregated condensates overlap over the length scale $\Lambda=\xi / \sqrt{a_{12} / \sqrt{a_{11} a_{22}}-1}$, where $\xi$ is the healing length [18]. For the JILA experiments on phase-segregated states $\Lambda \approx 47 \xi$. If, however, the penetration depth $\Lambda \ll R$, where $R$ is the size of the system, the condensates can be approximately treated as nonoverlapping, which we assume to be the case. The 
effect of the overlapping results in finite surface tension and can be included via boundary conditions at the interface. However, in the TF limit such effect is small, we shall discuss it in Sec. IV. If the condensates do not overlap one can neglect the last terms in Eqs. (1), (2). As a result, the dynamical equations for $\Psi_{1}$ and $\Psi_{2}$ decouple. However, the two condensate components are coupled by boundary conditions at the interface which require continuity of pressure and the normal velocity.

For definiteness we assume that stationary configuration is spherically symmetric with the central core dominated by the first component and an outer shell from the second species (see Fig. 1). The stationary density distribution $n_{i}=\left|\Psi_{i}\right|^{2}$ of two components is given by

$$
\begin{aligned}
& n_{1}=\frac{\mu_{1}}{G_{11}}\left(1-\frac{r^{2}}{R_{1}^{2}}\right), \quad 0<r<R_{*}, \\
& n_{2}=\frac{\mu_{2}}{G_{22}}\left(1-\frac{r^{2}}{R_{2}^{2}}\right), \quad R_{*}<r<R_{2},
\end{aligned}
$$

where $G_{i i}=4 \pi \hbar^{2} a_{i i} / m, R_{i}=\sqrt{2 \mu_{i} / m \omega_{0}^{2}}$. The normalization condition $\int n_{i} d V=N_{i}$, where $N_{i}$ are the numbers of condensate particles, determines the chemical potentials $\mu_{i}$. The position of the phase boundary $R_{*}$ is given by the condition that pressures exerted by both condensates are equal [29]:

$$
R_{*}=R_{2} \sqrt{\frac{1-\kappa \lambda}{(1-\kappa) \lambda}}
$$

where we have introduced dimensionless parameters

$$
\kappa=\sqrt{\frac{a_{11}}{a_{22}}}, \quad \lambda=\frac{\mu_{2}}{\mu_{1}} .
$$

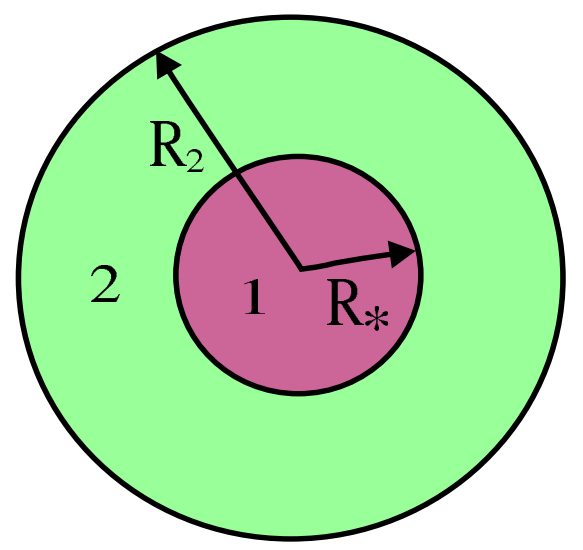

Fig. 1. Symmetric configuration of BEC components.

The symmetric configuration is favorable when $\kappa$ differs from unity, with the less repulsive component being in the middle $\left(a_{11}<a_{22}\right.$, that is $\left.\kappa<1\right)$. At the interface between the two components the stationary condensate densities are

$$
n_{1}=\frac{\kappa\left(\mu_{2}-\mu_{1}\right)}{G_{11}(1-\kappa)}, \quad n_{2}=\frac{\left(\mu_{2}-\mu_{1}\right)}{G_{22}(1-\kappa)} .
$$

$n_{2} / n_{1}=\kappa<1$.

One can rewrite the time dependent Gross-Pitaevskii equations in a hydrodynamic form which shows an analogy between our problem and the motion of two immiscible fluids. In the strong phase-segregated regime the dynamics of each components is described by the following hydrodynamic equations 


$$
\begin{gathered}
\frac{\partial n}{\partial t}+\nabla \cdot(n \mathbf{V})=0, \\
\frac{1}{2} m V^{2}+V_{\mathrm{tr}}-\frac{\hbar^{2}}{2 m} \frac{1}{\sqrt{n}} \Delta \sqrt{n}+G n+m \frac{\partial \Phi}{\partial t}=\mu,
\end{gathered}
$$

where $\mathbf{V}$ is the condensate velocity and $\Phi$ is the velocity potential, $\mathbf{V}=\nabla \Phi$. Eq. (9) is continuity equation for compressible flow, while Eq. (10) is the analog of the Bernoulli equation with $(e+P) / n=-\frac{\hbar^{2}}{2 m} \frac{1}{\sqrt{n}} \Delta \sqrt{n}+G n$, where $e$ is the internal energy density and $P$ is the pressure. The kinetic energy (quantum) pressure is omitted in the TF limit and, therefore, $e=G n^{2} / 2$. As a result, from Eq. (10) we obtain for the pressure

$$
2 P=G n^{2}=\mu n-n V_{\mathrm{tr}}-m n \frac{\partial \Phi}{\partial t}-\frac{1}{2} m n V^{2} .
$$

The trapping potential plays the role of gravitational potential in hydrodynamics. The linearized hydrodynamic equations determine the condensate normal modes. In terms of perturbation in the condensate density $n^{\prime}$ and the velocity potential $\Phi$ the normal-mode amplitudes satisfy the coupled equations 30]

$$
\begin{gathered}
i \omega n^{\prime}=\nabla \cdot\left(\mathbf{V} n^{\prime}\right)+\nabla \cdot(n \nabla \Phi), \\
i \omega \Phi=\mathbf{V} \cdot \nabla \Phi+\frac{G}{m} n^{\prime}-\frac{\hbar^{2}}{4 m^{2} n} \nabla \cdot\left[n \nabla\left(\frac{n^{\prime}}{n}\right)\right],
\end{gathered}
$$

where $n$ is the static condensate density and $\mathbf{V}$ is the static condensate velocity. In our problem $\mathbf{V}=\mathbf{0}$, hence, $n^{\prime} \approx i \omega m \Phi / G$ and the equation for the perturbation in velocity potential is 31

$$
\frac{2 \omega^{2}}{\omega_{0}^{2}} \Phi-2 r \frac{\partial \Phi}{\partial r}+\left(R^{2}-r^{2}\right) \Delta \Phi=0
$$

One can seek solution of this equation in the form

$$
\Phi=\Phi(r) Y_{l m}(\theta, \phi),
$$

where $Y_{l m}(\theta, \phi)$ is the spherical harmonics. Then, using $\Delta Y_{l m}(\theta, \phi)=-l(l+1) / r^{2}$, we obtain the following equation for the radial function $\Phi(r)$

$$
r^{2}\left(r^{2}-R^{2}\right) \frac{d^{2} \Phi}{d r^{2}}+\left(4 r^{2}-2 R^{2}\right) r \frac{d \Phi}{d r}+\left[l(l+1) R^{2}-r^{2}\left(\frac{2 \omega^{2}}{\omega_{0}^{2}}+l(l+1)\right)\right] \Phi=0 .
$$

Let us derive boundary conditions for $\Phi$ at the interface. The change in density at the interface is the sum of the term from the movement of the interface and that from the perturbation of the density. Let $\varsigma=\varsigma(\theta, \phi, t)$ be a small deviation of the $r$-coordinate of the interphase from its stationary value $R_{*}$. Then the condensate density at the interface is $n\left(R_{*}+\varsigma, t\right) \approx n\left(R_{*}, t\right)+\varsigma \partial n\left(R_{*}, t\right) / \partial r$, and, hence, the pressure is given by

$$
P=\frac{G n^{2}}{2} \approx \frac{G n^{2}\left(R_{*}, t\right)}{2}+G n\left(R_{*}, t\right) \frac{\partial n\left(R_{*}, t\right)}{\partial r} \varsigma .
$$

In terms of the density perturbation $n^{\prime}, n\left(R_{*}, t\right)=n\left(R_{*}\right)+n^{\prime}\left(R_{*}, t\right)$, we obtain

$$
P \approx \frac{G n^{2}\left(R_{*}\right)}{2}+G n\left(R_{*}\right) n^{\prime}\left(R_{*}, t\right)-n\left(R_{*}\right) m \omega_{0}^{2} R_{*} \varsigma .
$$

Continuity of the pressure at the interface results in the following boundary condition

$$
G_{11} n_{1}\left(R_{*}\right) n_{1}^{\prime}\left(R_{*}, t\right)-n_{1}\left(R_{*}\right) m \omega_{0}^{2} R_{*} \varsigma_{1}=G_{22} n_{2}\left(R_{*}\right) n_{2}^{\prime}\left(R_{*}, t\right)-n_{2}\left(R_{*}\right) m \omega_{0}^{2} R_{*} \varsigma_{2}
$$

Taking into account $G n^{\prime}=i m \omega \Phi$ and $\partial \varsigma / \partial t=V_{r}=\partial \Phi / \partial r$ we finally obtain the following boundary condition for the velocity potential at the interface

$$
\omega_{0}^{2} R_{*} \frac{\partial \Phi_{1}}{\partial r}-\omega^{2} \Phi_{1}=\kappa \omega_{0}^{2} R_{*} \frac{\partial \Phi_{2}}{\partial r}-\kappa \omega^{2} \Phi_{2}
$$

Continuity of $V_{r}$ gives another boundary condition

$$
\frac{\partial \Phi_{1}}{\partial r}=\frac{\partial \Phi_{2}}{\partial r} .
$$

Eq. (15) and the boundary conditions (18), (19) compose a complete set of equations necessary to determine normal modes of the system. 


\section{NORMAL MODES}

For one component condensates the solutions of Eq. (15) which are finite at $r=0$ and $r=R$ are

$$
\Phi(r) \propto r^{l} P_{n}^{\left(l+\frac{1}{2}, 0\right)}\left(1-\frac{2 r^{2}}{R^{2}}\right)
$$

where $P_{n}^{\left(l+\frac{1}{2}, 0\right)}(x)$ are Jacobi polynomials, $n$ is the radial quantum number. The corresponding eigenfrequencies are given by [31]

$$
\omega^{2}=\omega_{0}^{2}(l+n(2 n+2 l+3))
$$

For the two component condensate the normal mode frequencies are different from the general case and new modes appear. However, we shall show that frequencies corresponding to $n=0$ remain the same as for the one component system. Rewriting the function $\Phi=r^{l} \eta\left(r^{2} / R^{2}\right)$ in Eq. 15) results in a hypergeometric equation for $\eta(\xi)$

$$
\xi(\xi-1) \eta_{\xi \xi}^{\prime \prime}+\left[\left(l+\frac{5}{2}\right) \xi-l-\frac{3}{2}\right] \eta_{\xi}^{\prime}+\frac{1}{2}\left(l-\frac{\omega^{2}}{\omega_{0}^{2}}\right) \eta=0 .
$$

Hence, general solutions for the inner and outer condensates which are regular at $r=0$ and $r=R_{2}$ respectively have the form

$$
\Phi_{1}=C_{1} r^{l} F\left(\alpha, \beta, l+3 / 2, \frac{r^{2}}{R_{1}^{2}}\right), \quad \Phi_{2}=C_{2} r^{l} F\left(\alpha, \beta, 1,1-\frac{r^{2}}{R_{2}^{2}}\right),
$$

where $F$ is hypergeometric function, $C_{1}, C_{2}$ are constants and

$$
\alpha=\frac{1}{2}\left[l+\frac{3}{2}-\sqrt{l^{2}+l+\frac{9}{4}+\frac{2 \omega^{2}}{\omega_{0}^{2}}}\right], \quad \beta=\frac{1}{2}\left[l+\frac{3}{2}+\sqrt{l^{2}+l+\frac{9}{4}+\frac{2 \omega^{2}}{\omega_{0}^{2}}}\right] .
$$

Using Eq. (23), the boundary conditions (18), (19) and the mathematical identity

$$
\frac{d}{d z} F(\alpha, \beta, \gamma, z)=\frac{\alpha \beta}{\gamma} F(\alpha+1, \beta+1, \gamma+1, z)
$$

we obtain the equation for the normal mode frequencies

$$
\frac{\omega^{2}}{\omega_{0}^{2}}=(1-\kappa) \frac{\left[l(l+3 / 2)+\left(l-\omega^{2} / \omega_{0}^{2}\right) \lambda x s_{1}(\omega, x)\right]\left[\left(l-\omega^{2} / \omega_{0}^{2}\right) x s_{2}(\omega, x)-l\right]}{\left[l(l+3 / 2)(\kappa-1)+x\left(l-\omega^{2} / \omega_{0}^{2}\right)\left(\kappa \lambda s_{1}(\omega, x)+(l+3 / 2) s_{2}(\omega, x)\right)\right]},
$$

where

$$
s_{1}(\omega, x)=\frac{F(\alpha+1, \beta+1, l+5 / 2, \lambda x)}{F(\alpha, \beta, l+3 / 2, \lambda x)}, \quad s_{2}(\omega, x)=\frac{F(\alpha+1, \beta+1,2,1-x)}{F(\alpha, \beta, 1,1-x)}, \quad x=\frac{R_{*}^{2}}{R_{2}^{2}} .
$$

One of the solutions of Eq. (24) is $\omega^{2}=l \omega_{0}^{2}$, which coincide with those for one component condensate. For this solution the components oscillate in-phase and $\Phi_{1}=\kappa \Phi_{2} \propto r^{l} Y_{l m}(\theta, \phi)$. Another exact solution is $\omega^{2}=5 \omega_{0}^{2}$ with $\Phi_{1} \propto 1-5 r^{2} / 3 R_{1}^{2}, \Phi_{2} \propto \lambda\left(1-5 r^{2} / 3 R_{2}^{2}\right)$ which corresponds to $l=0, n=1$. For this solution the components oscillate out-of-phase if $\sqrt{3 / 5} R_{1}<R_{*}<\sqrt{3 / 5} R_{2}$ and in-phase otherwise.

\section{A. Numerical results}

We solve Eq. (24) numerically and find normal mode frequencies $\omega$ of the two component condensate as a function of the parameter $\kappa=\sqrt{a_{11} / a_{22}}$ for different fixed ratios $R_{*} / R_{2}$. The ratio $R_{*} / R_{2}$ can be directly measured experimentally. Fig. 2 shows the normal mode frequencies for $l=0$. At fixed $l$ there is an infinite number of branches which correspond to different radial quantum numbers $n$. We plot only the lowest modes with $n=0,1,2$. In Fig. 3 the normal modes are estimated for $l=1$. In the limiting case $a_{11}=a_{22}(\kappa=1)$ the condensates behave as an one component system and the condensate modes coincide with Stringari's result (21). Fig. 4 shows the low frequency 
modes that become imaginary at $\kappa>1$. These modes are peculiar for two component systems and are analogous to the waves at the interface between two layers of immiscible fluids under gravity [32]. In trapped condensates the gradient of trap potential plays the role of gravitational field. As soon as $\kappa$ becomes greater than 1, which means $n_{2}>n_{1}$ at the interface, the system becomes unstable the same way as two immiscible fluids in gravitational field when the more dense layer is on the top. One should mention that normal modes with imaginary frequency also appear at the transition point from a binary mixture into a phase separated state which occurs at $a_{12}=\sqrt{a_{11} a_{22}}$ [19,23,27. In that case, the fastest decaying mode occurs at a finite wavevector, resulting in a quantum spinodal transition 33.

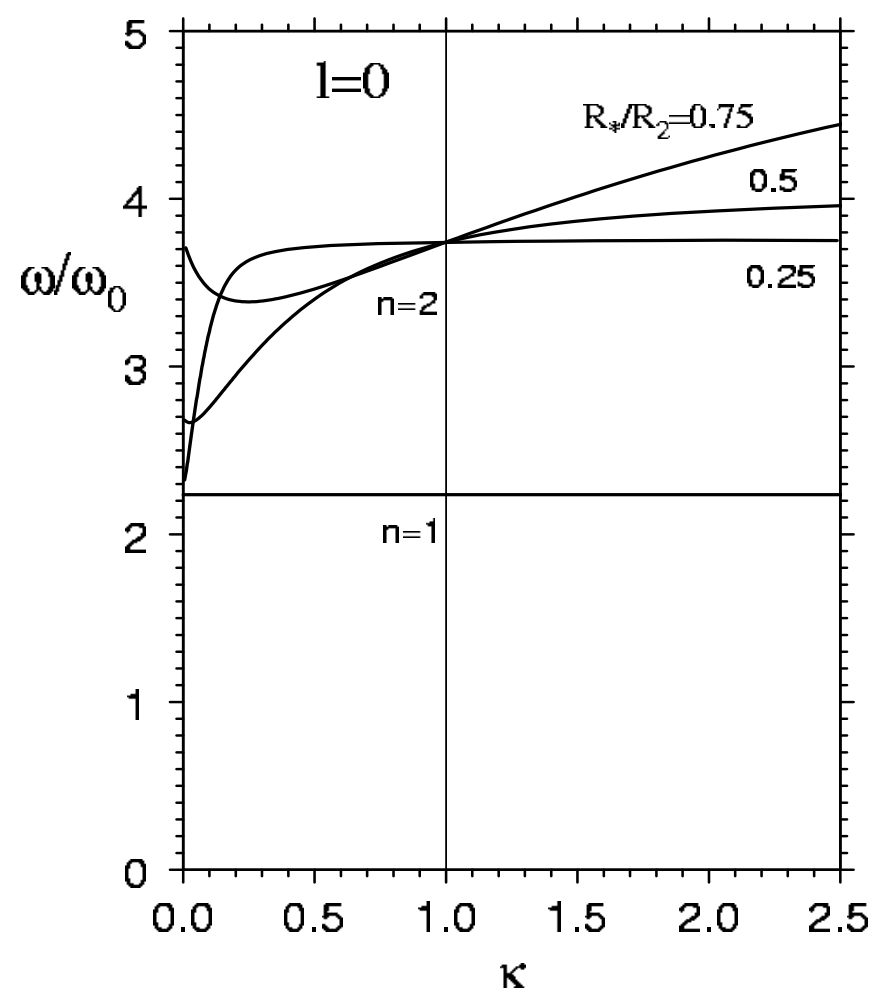

Fig. 2. Normal mode frequencies for $l=0$ and $n=1,2$ as a function of $\kappa=\sqrt{a_{11} / a_{22}}$. The mode with $n=2$ depends on position of the interface, the mode frequency is estimated for $R_{*} / R_{2}=0.25,0.5,0.75$. For $\kappa=1$ the mode frequencies coincide with those for the one component condensate. 


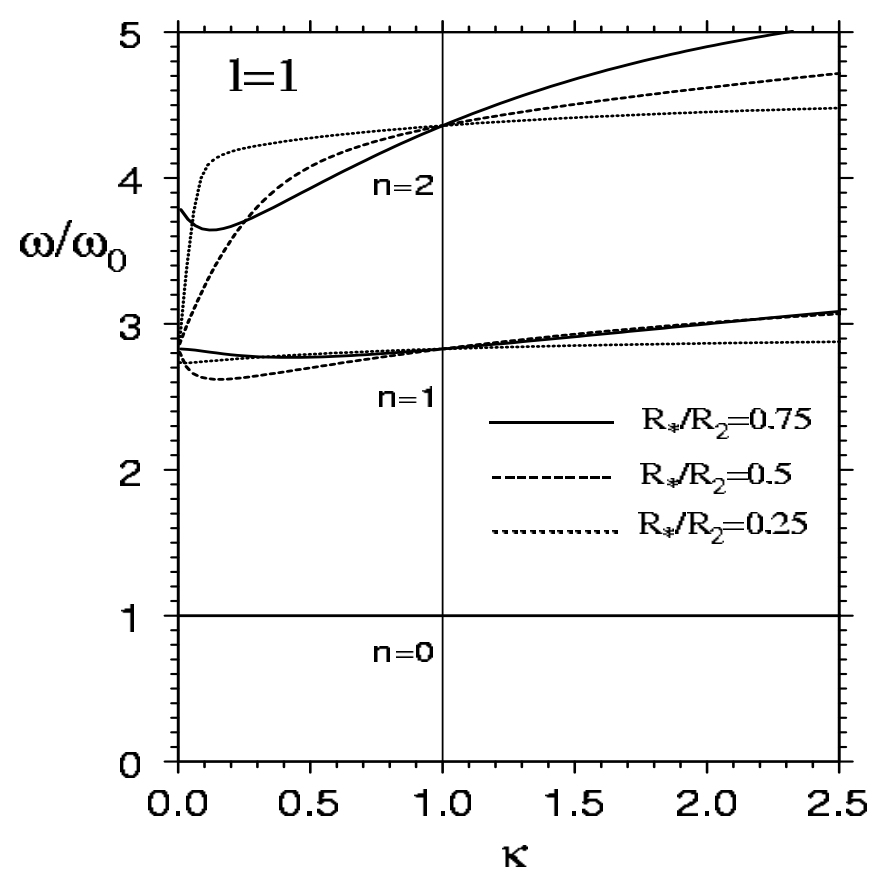

Fig. 3. Normal mode frequencies for $l=1$ and $n=0,1,2$.

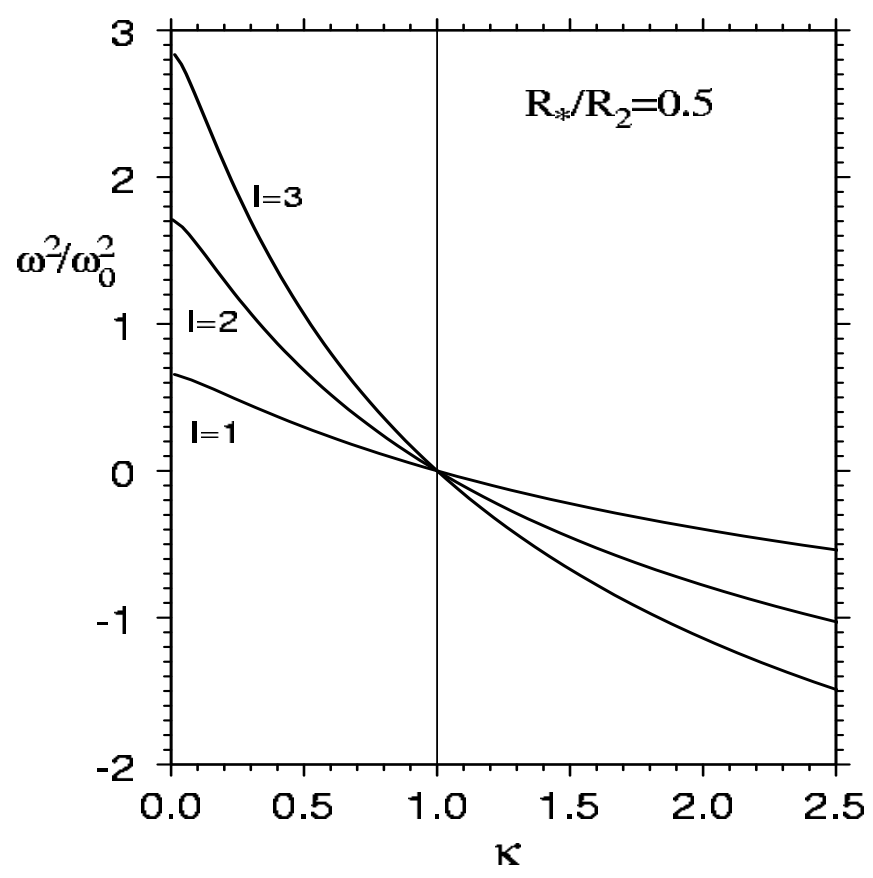

Fig. 4. Low frequency modes as a function of $\kappa=\sqrt{a_{11} / a_{22}}$ for different $l=1,2,3$. The position of the interface is $R_{*}=R_{2} / 2$. Frequencies become imaginary at $\kappa>1$.

\section{B. Low frequency modes}

Here we study the low frequency modes in detail. In the region $|1-\kappa| \ll 1$ the mode frequencies are small: $|\omega| \ll \omega_{0}$. In this limit one can take $\omega=0$ in the right side of Eq. (24) and put $\kappa, \lambda \approx 1$ in the multiple. As a result, we obtain 


$$
\omega^{2} \approx \omega_{0}^{2}(1-\kappa) f(l, x)
$$

where

$$
f(l, x)=\frac{l\left(l+3 / 2+x s_{1}(0, x)\right)\left(x s_{2}(0, x)-1\right)}{x\left[s_{1}(0, x)+(l+3 / 2) s_{2}(0, x)\right]} .
$$

Eq. (25) describes behavior of the low frequency modes in the region close to the point of instability $\kappa=1$. In this region $\omega \propto \sqrt{1-\kappa}$ and becomes imaginary when $\kappa>1$. In Fig. 5 we plot $f$ as a function of $x=R_{*} / R_{2}$ for different $l$. Imaginary part of frequencies is greater for larger $l$ and decreases with increasing $R_{*} / R_{2}$, that is bigger inner droplets are more stable. Figs. 6, 7 show the radial distribution of the density perturbation for the low frequency modes with different $l$. In our estimates we choose the radius of the inner droplet $R_{*}=R_{2} / 2$. The density perturbations are normalized so that $n_{2}^{\prime}\left(R_{2}\right)=1$. For the low frequency modes the two condensates oscillate out-of-phase. Fig. 6 corresponds to $\kappa=0.9$ (stable region), while for Fig. $7 \kappa=1.1$ (unstable regime). One can see that the normal mode profile undergoes no changes at the point of phase transition, although the mode frequencies become imaginary at this point. However, due to the relation $n^{\prime} \approx i \omega m \Phi / G$, the phase shift between oscillations of $n^{\prime}$ and $\Phi$ changes at the instability point from $\pi / 2$ to 0 . Modes with small $l$ are delocalized and the whole condensate is involved in oscillations. With increasing $l$, however, the modes become localized near the interface and in the limit $l \rightarrow \infty$ they are similar to gravitational waves at the surface of deep water with the dispersion relation $\omega \propto \sqrt{k}$.

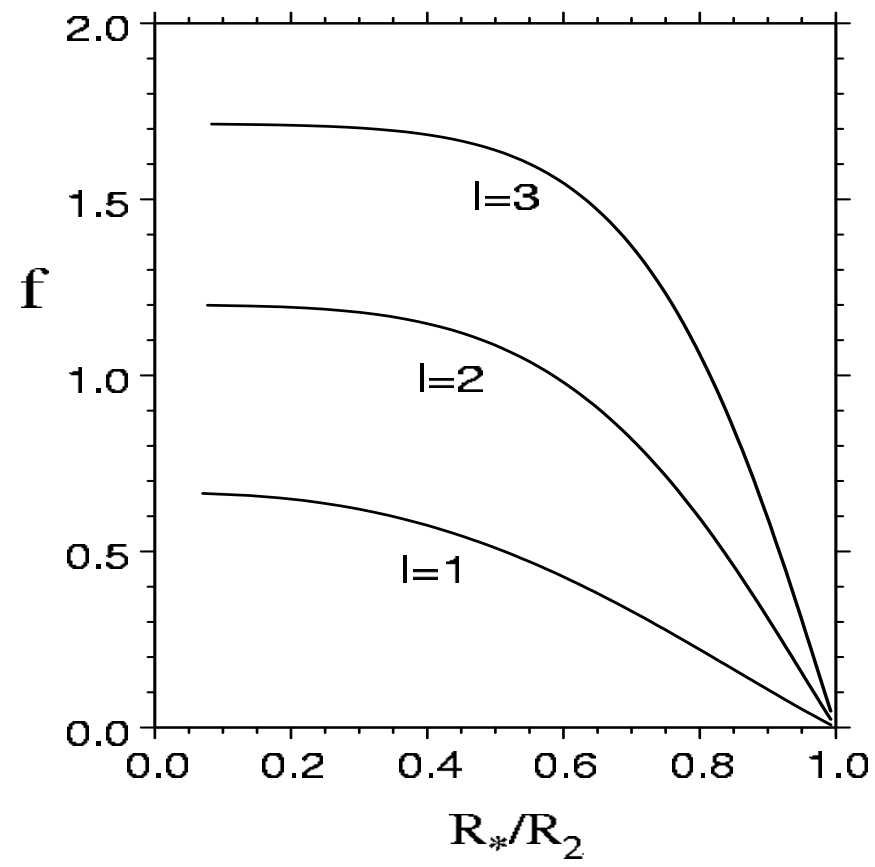

Fig. 5. Dependence of the factor $f$ in Eq. (25) on the position of the interface $R_{*} / R_{2}$ for modes with different $l=1,2,3$. 


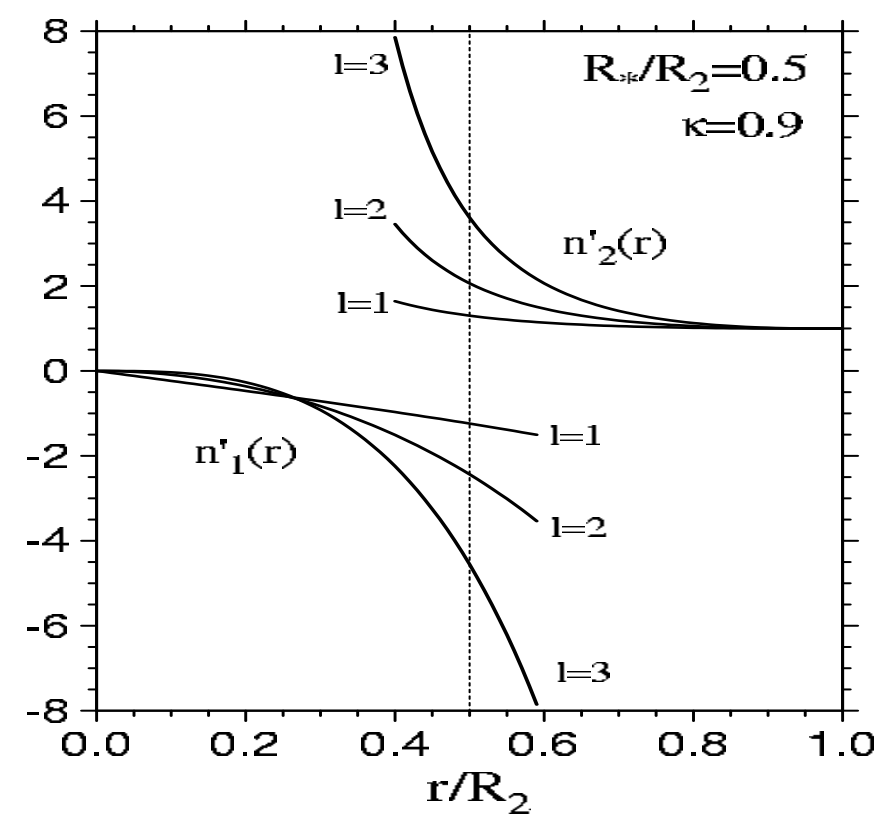

Fig. 6. Perturbation in the condensate density of the two components $n_{1}^{\prime}(r)$ and $n_{2}^{\prime}(r)$ for the low frequency modes with different $l=1,2,3$. The position of the interface is $R_{*}=R_{2} / 2$ (dash line) and $\kappa=0.9$ (stable region).

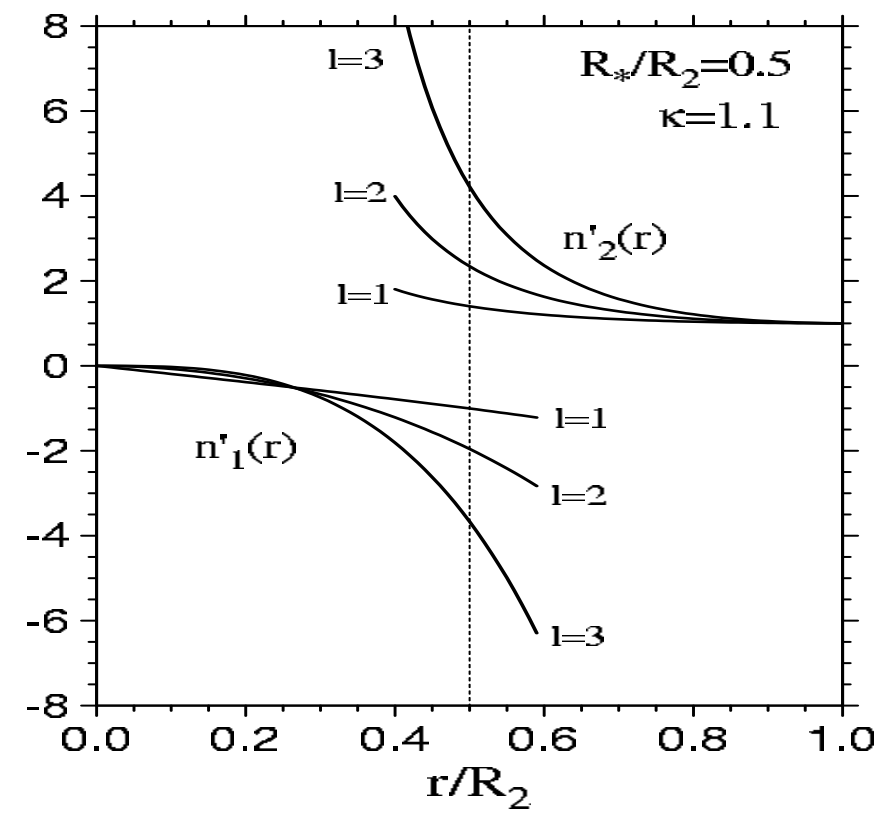

Fig. 7. The same as in Fig. 6, but for $\kappa=1.1$ (unstable region).

\section{EFFECT OF INTERFACE TENSION}

Interface tension is small in the TF limit and results in corrections of the order of $\xi / R_{2}$ to the normal mode frequencies. However, for the low frequency modes in the vicinity $\kappa \approx 1$ the effect of the interface tension is substantial because the mode frequencies themselves are close to zero. The surface tension $\sigma$ modifies the boundary condition for the pressure so that the pressure difference at the interface is equal to the surface tension pressure $P_{1}-P_{2}=$ $\sigma\left(1 / r_{1}+1 / r_{2}\right)$, where $r_{1}, r_{2}$ are the principle radii of curvature. The stationary position of the interface is determined 
by the equation

$$
\frac{G_{11} n_{1}^{2}}{2}=\frac{G_{22} n_{2}^{2}}{2}+\frac{2 \sigma}{R_{*}}
$$

which results in the following ratio of densities at the interface

$$
\frac{n_{2}}{n_{1}}=\sqrt{\kappa^{2}-\frac{4 \sigma}{G_{22} n_{1}^{2} R_{*}}}=\kappa_{\mathrm{eff}}
$$

The interface shape oscillates during the condensate motion which produces oscillations of the surface pressure. For interface shape close to spherical 34

$$
\frac{1}{r_{1}}+\frac{1}{r_{2}}=\frac{2}{R_{*}}-\frac{2 \varsigma}{R_{*}^{2}}-\frac{\Delta_{\theta, \phi} \varsigma}{R_{*}^{2}}
$$

where $\Delta_{\theta, \phi}$ is the angular part of Laplace's operator. Also one should take into account that $\sigma$ itself depends on local density and varies during the interface motion. We assume $\sigma \propto \sqrt{\frac{1}{G_{11} n_{1}}+\frac{1}{G_{22} n_{2}}} n_{1} n_{2}$ [19]. Then, taking into account $n\left(R_{*}+\varsigma, t\right) \approx n\left(R_{*}\right)+n^{\prime}\left(R_{*}, t\right)+\varsigma \partial n\left(R_{*}\right) / \partial r$, we obtain

$$
\sigma=\sigma_{0}\left(1+\frac{m}{G_{11} n_{1}}\left(i \omega \Phi_{1}-\omega_{0}^{2} R_{*} \varsigma\right)\left[\frac{1+2 \kappa_{\mathrm{eff}}}{2\left(1+\kappa_{\mathrm{eff}}\right)}\right]+\frac{m}{G_{22} n_{2}}\left(i \omega \Phi_{2}-\omega_{0}^{2} R_{*} \varsigma\right)\left[\frac{2+\kappa_{\mathrm{eff}}}{2\left(1+\kappa_{\mathrm{eff}}\right)}\right]\right),
$$

where $\sigma_{0}$ is the tension for the stationary configuration. As a result, the boundary condition (18) contains extra terms and becomes

$$
\begin{gathered}
\left(m n_{1}-\frac{2 m \sigma_{0}}{R_{*} G_{11} n_{1}}\left[\frac{1+2 \kappa_{\mathrm{eff}}}{2\left(1+\kappa_{\mathrm{eff}}\right)}\right]\right)\left[\omega_{0}^{2} R_{*} \frac{\partial \Phi_{1}}{\partial r}-\omega^{2} \Phi_{1}\right]-\frac{\sigma_{0}}{R_{*}^{2}}\left(2 \frac{\partial \Phi_{1}}{\partial r}+\Delta_{\theta, \phi} \frac{\partial \Phi_{1}}{\partial r}\right)= \\
=\left(m n_{2}+\frac{2 m \sigma_{0}}{R_{*} G_{22} n_{2}}\left[\frac{2+\kappa_{\mathrm{eff}}}{2\left(1+\kappa_{\mathrm{eff}}\right)}\right]\right)\left[\omega_{0}^{2} R_{*} \frac{\partial \Phi_{2}}{\partial r}-\omega^{2} \Phi_{2}\right],
\end{gathered}
$$

while Eq. (19) remains the same. For the radial part of the velocity potential we obtain

$$
\begin{gathered}
\left(1-\frac{\sigma_{0}}{R_{*} P}\left[\frac{1+2 \kappa_{\mathrm{eff}}}{2\left(1+\kappa_{\mathrm{eff}}\right)}\right]\right)\left[\omega_{0}^{2} R_{*} \frac{\partial \Phi_{1}}{\partial r}-\omega^{2} \Phi_{1}\right]+\frac{\sigma_{0}(l-1)(l+2)}{m n_{1} R_{*}^{2}} \frac{\partial \Phi_{1}}{\partial r}= \\
=\kappa_{\mathrm{eff}}\left(1+\frac{\sigma_{0}}{R_{*} P}\left[\frac{2+\kappa_{\mathrm{eff}}}{2\left(1+\kappa_{\mathrm{eff}}\right)}\right]\right)\left[\omega_{0}^{2} R_{*} \frac{\partial \Phi_{2}}{\partial r}-\omega^{2} \Phi_{2}\right]
\end{gathered}
$$

where $P$ is the pressure at the interface. The modified boundary condition results in the following equation for the low frequency modes

$$
\omega^{2} \approx \omega_{0}^{2}\left(1-\frac{3 \sigma_{0}}{2 R_{*} P}+\frac{\sigma_{0}(l-1)(l+2)}{m n_{1} \omega_{0}^{2} R_{*}^{3}}-\kappa_{\mathrm{eff}}\right) f .
$$

where $n_{1}$ is the density at the interface. The interface tension shifts the frequencies of the lowest modes and narrows the stability region. For $l=1$ the inner droplet moves as a whole without changing its shape. However, the displacement of the droplet into the less dense region decreases the interface energy which is proportional to $n^{3 / 2}$. This is the origin for the contribution $-3 \sigma_{0} / R_{*} P$ to the mode frequency. The mode frequencies now become imaginary at

$$
\kappa^{2}>1-\frac{\sigma_{0}}{R_{*} P}+\frac{2 \sigma_{0}(l-1)(l+2)}{m n_{1} \omega_{0}^{2} R_{*}^{3}} .
$$

As $\kappa$ increases the mode with $l=1$ becomes imaginary first which determines the system's stability limit. When the surface tension is absent, the higher $l$ is the more unstable the mode is. In the present case, the two component condensate is locally unstable when

$$
\frac{a_{11}}{a_{22}}>1-\frac{\sigma_{0}}{R_{*} P}
$$




\section{GLOBAL STABILITY: ENERGY OF ASYMMETRIC PHASE}

So far we have discussed the local stability of the symmetric phase. We next turn our attention to its global stability by comparing the energies of the symmetric phase and the asymmetric phase. Let us consider an asymmetric state in which component 1 sits on the top of the component 2. The transition occurs when $\kappa$ is close to 1 which we assume. Hence, the interface is approximately flat (see Fig. 8).

\section{symmetric}

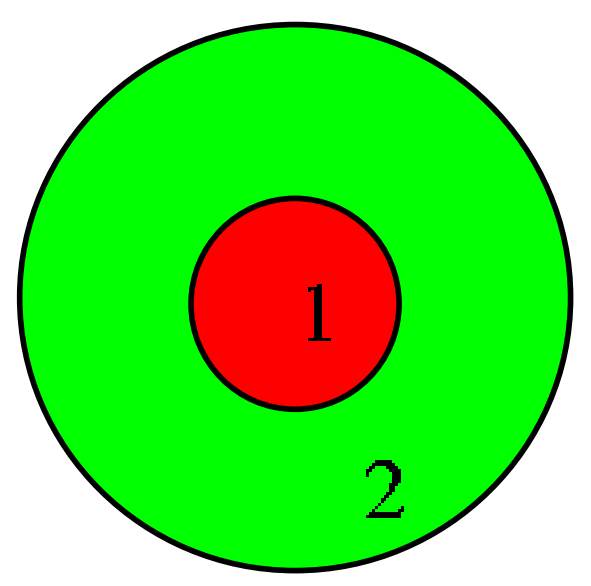

asymmetric

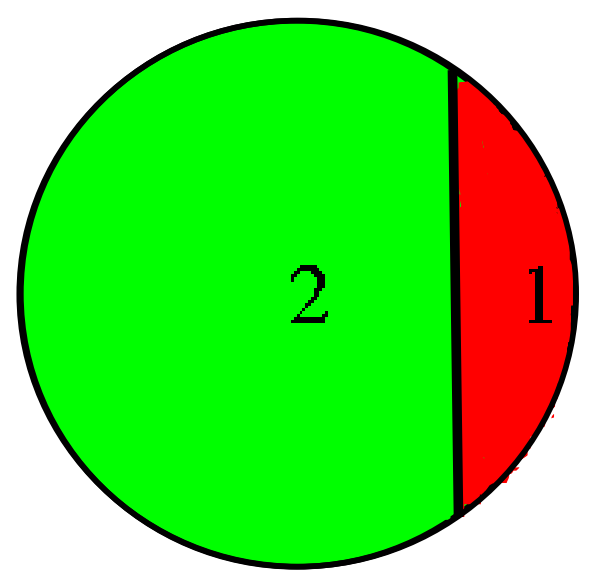

Fig. 8. Symmetric and Asymmetric phases of two component BECs.

In the Thomas-Fermi approximation, the density distribution is still given by Eqs. (4) and (5), but the interface position is now different. The TF density distribution suggests that the outer edge of the condensates has a spherical shape and, therefore, in the asymmetric configuration the condensates have equal chemical potentials $\mu_{1 a}=\mu_{2 a}=\mu$. Carrying out the integration, we find that the energy of the asymmetric state is given by

$$
E_{a}=\frac{\mu}{2}\left(N_{1}+N_{2}\right)+\frac{\pi m^{2} \omega_{0}^{4} R^{7}}{8}\left(\frac{1}{G_{11}}-\frac{1}{G_{22}}\right)\left[\frac{4}{35}-\frac{d}{6 R}+\frac{d^{5}}{10 R^{5}}-\frac{d^{7}}{21 R^{7}}\right]+\frac{\pi m^{2} \omega_{0}^{4} R^{7}}{35 G_{22}}+E_{s a},
$$

where $z=d$ is the position of the interface, $E_{s a}$ is the interface energy and $R=\sqrt{2 \mu / m \omega_{0}^{2}}$ is the radius of the sphere. The radius $R$ (and hence $\mu$ ) and the position $d$ are determined by the number of particles

$$
\begin{aligned}
& N_{1}=\frac{\pi \mu R^{3}}{15 G_{11}}\left[4-\frac{15 d}{2 R}+\frac{5 d^{3}}{R^{3}}-\frac{3 d^{5}}{2 R^{5}}\right], \\
& N_{2}=\frac{\pi \mu R^{3}}{15 G_{22}}\left[4+\frac{15 d}{2 R}-\frac{5 d^{3}}{R^{3}}+\frac{3 d^{5}}{2 R^{5}}\right] .
\end{aligned}
$$

From Eqs. (33), (34), we obtain

$$
G_{11} N_{1}+G_{22} N_{2}=\frac{4 \pi m \omega_{0}^{2} R^{5}}{15}
$$

which determines $R$. The position of the interface can be found from

$$
4-\frac{15 d}{2 R}+\frac{5 d^{3}}{R^{3}}-\frac{3 d^{5}}{2 R^{5}}=\frac{8 G_{11} N_{1}}{G_{22} N_{2}+G_{11} N_{1}} .
$$

The energy of a spherically symmetric configuration when the first droplet is located inside and the second outside is given by

$$
E_{0}=\frac{\mu_{1} N_{1}}{2}+\frac{\mu_{2} N_{2}}{2}+\pi m \omega_{0}^{2}\left[\left(\frac{\mu_{1}}{G_{11}}-\frac{\mu_{2}}{G_{22}}\right) \frac{R_{*}^{5}}{5}+\frac{m \omega_{0}^{2}}{2}\left(\frac{1}{G_{22}}-\frac{1}{G_{11}}\right) \frac{R_{*}^{7}}{7}+\right.
$$




$$
\left.+\frac{2^{7 / 2} \mu_{2}^{7 / 2}}{35 G_{22} m^{5 / 2} \omega_{0}^{5}}\right]+E_{s 0}
$$

where $E_{s 0}$ is the interface energy in the symmetric state.

When the system is close to the point of phase transition the interface energies of two configurations are given by (for details see Sec. VI)

$$
\begin{aligned}
& E_{s 0}=\frac{8 \pi \hbar R_{*}^{2} \mu^{3 / 2}}{\sqrt{3 m} G} \sqrt{a_{12} / \sqrt{a_{11} a_{22}}-1}\left(1-\frac{R_{*}^{2}}{R^{2}}\right)^{3 / 2}, \\
& E_{s a}=\frac{4 \pi \hbar R^{2} \mu^{3 / 2}}{5 \sqrt{3 m} G} \sqrt{a_{12} / \sqrt{a_{11} a_{22}}-1}\left(1-\frac{d^{2}}{R^{2}}\right)^{5 / 2} .
\end{aligned}
$$

In general, these expressions need to be numerically evaluated. They are analytically tractable in the limit when one of the components is particularly small, to which we turn our attention next.

\section{A. Energy for $N_{1} \ll N_{2}$}

If the number of atoms of the first species is much less than $N_{2}$ then $R_{*} \ll R, d / R \approx 1-\left(4 N_{1} / 5 N_{2}\right)^{1 / 3}$ and from Eqs. (38), (39) we find

$$
\begin{gathered}
E_{s 0} \approx \frac{8 \pi \hbar R_{*}^{2} \mu^{3 / 2}}{\sqrt{3 m} G} \sqrt{a_{12} / \sqrt{a_{11} a_{22}}-1}, \\
E_{s a} \approx 1.008 \sqrt{\frac{R_{*}}{R}} E_{s 0} \ll E_{s 0} .
\end{gathered}
$$

The interface energy of the asymmetric state is negligible. Close to the point of phase transition the energy difference between the asymmetric and symmetric configuration is given by

$$
E_{a}-E_{0}=\frac{1}{2} \mu N_{1}\left[(1-\kappa)^{2}-\frac{4 \sqrt{6} \xi}{R_{*}} \sqrt{a_{12} / \sqrt{a_{11} a_{22}}-1}\right],
$$

where $\xi=\hbar / \sqrt{2 m \mu}$.

In the absence of the interface tension $(\xi \rightarrow 0)$ the asymmetric configuration has greater energy no matter what is the value of $\kappa$ in comparison with 1 . However, the interface tension makes the asymmetric phase preferable at some $\kappa$ and results in symmetric-asymmetric phase transition. The condition $E_{a}=E_{0}$ describes a line of global instability of the system

$$
\frac{a_{11}}{a_{22}}=1-4\left(\frac{\sqrt{6} \xi}{R_{*}}\right)^{1 / 2}\left(a_{12} / \sqrt{a_{11} a_{22}}-1\right)^{1 / 4} .
$$

The soft mode condition gives the line of local stability

$$
\frac{a_{11}}{a_{22}}=1-\frac{4 \sqrt{6} \xi}{3 R_{*}}\left(a_{12} / \sqrt{a_{11} a_{22}}-1\right)^{1 / 2} .
$$

In Fig. 9 we plot the phase diagram that shows different stability regions of two component condensates. In estimates we take $\xi / R_{*}=0.01$. When $a_{12}<\sqrt{a_{11} a_{22}}$ the homogeneous binary mixture is a stable state. Otherwise the two components are phase separated. In the later case for small ratio $a_{11} / a_{22}$ the state $(1,2)$ with the first component being inside and the second outside is the only stable configuration. If the ratio $a_{11} / a_{22}$ increases the configuration becomes globally unstable when we cross the left (solid) curve. However, the system is locally stable since the configuration corresponds to a local minima of energy. Further increase of $a_{11} / a_{22}$ crosses the line of local stability (another solid line) and the system undergoes a phase transition into a new stable asymmetric state. If initially the 
system is prepared in the $(2,1)$ state then with decreasing the ratio $a_{11} / a_{22}$ the configuration first becomes globally unstable when we cross the right dotted line and locally unstable when $a_{11} / a_{22}$ is close enough to 1 .

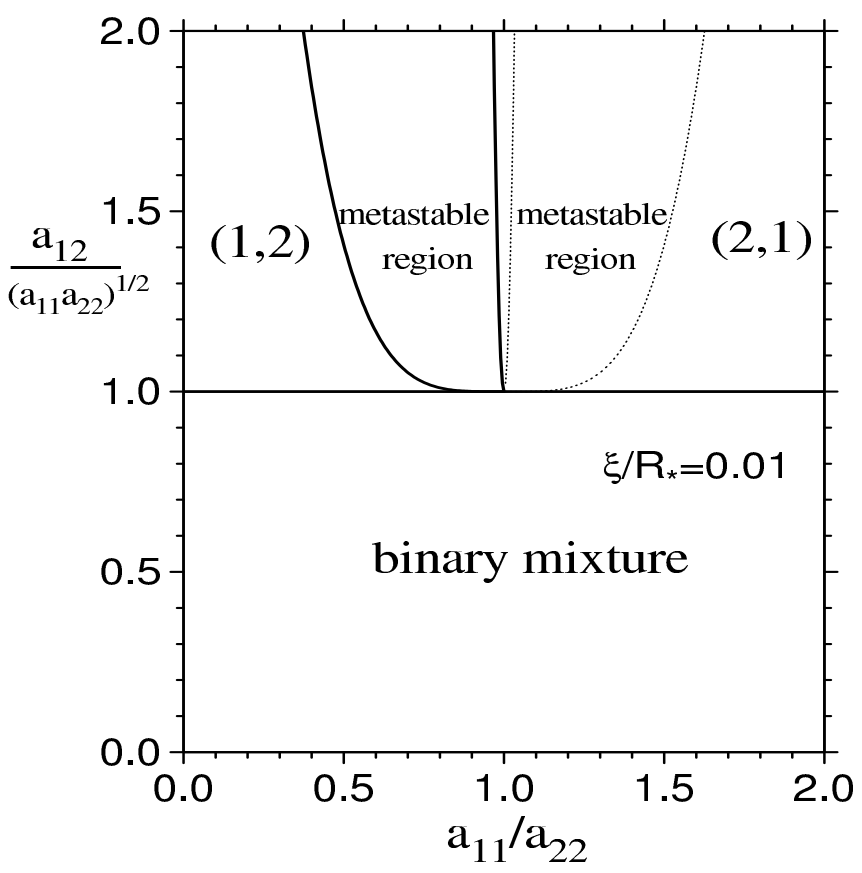

Fig. 9. Phase diagram of the two component condensate in coordinates displaying relative interaction strength between bosons.

\section{ENERGY AS A FUNCTION OF DROPLET DISPLACEMENT}

The question about the system's global stability is related to the energy of the state. To further explore the metastability of the system here we estimate the energy of the system for a displaced inner droplet. We assume the inner droplet has a spherical shape with a radius $r_{0}$ and the center of the sphere is displaced a distance $z_{0}$ from the trap center. The radius $r_{0}$ depends on $z_{0}$. The condensate densities are given by the same TF expressions (4), (5), however, the position of the interface is now different. We assume the number of particles of each species are fixed:

$$
\begin{gathered}
N_{1}=\frac{4 \pi}{3 G_{11}}\left[\mu_{1} r_{0}^{3}-\frac{3 m \omega_{0}^{2} r_{0}^{5}}{10}-\frac{m \omega_{0}^{2} r_{0}^{3} z_{0}^{2}}{2}\right], \\
N_{2}=\frac{4 \pi}{3} \frac{1}{G_{22}}\left[\frac{2}{5} \mu_{2} R_{2}^{3}-\mu_{2} r_{0}^{3}+\frac{3 m \omega_{0}^{2} r_{0}^{5}}{10}+\frac{m \omega_{0}^{2} r_{0}^{3} z_{0}^{2}}{2}\right] .
\end{gathered}
$$

For fixed numbers of particles, the chemical potentials are functions of the positions of the droplets and are not constants. In the TF limit we find for the energy of the system

$$
\begin{gathered}
E=\frac{\mu_{1} N_{1}}{2}+\frac{\mu_{2} N_{2}}{2}+\pi m \omega_{0}^{2}\left[\left(\frac{\mu_{1}}{G_{11}}-\frac{\mu_{2}}{G_{22}}\right)\left(\frac{r_{0}^{5}}{5}+\frac{r_{0}^{3} z_{0}^{2}}{3}\right)+\frac{m \omega_{0}^{2}}{2}\left(\frac{1}{G_{22}}-\frac{1}{G_{11}}\right)\left[\frac{r_{0}^{7}}{7}+\frac{2 r_{0}^{5} z_{0}^{2}}{3}+\frac{r_{0}^{3} z_{0}^{4}}{3}\right]+\right. \\
\left.+\frac{2^{7 / 2} \mu_{2}^{7 / 2}}{35 G_{22} m^{5 / 2} \omega_{0}^{5}}\right]+E_{s},
\end{gathered}
$$

where $E_{s}$ is the interface energy. The energy (46) depends on four parameters: $\mu_{1}, \mu_{2}, z_{0}, r_{0}$. However, only two parameters are independent because there are two restrictions imposed by particle conservation (44), 445). For 
simplicity let us consider the path for which $\mu_{2}=$ const. Then only one parameter is independent. If we choose $r_{0}$ as an independent variable then the energy as a function of $r_{0}$ can be expressed in an explicit form

$$
\begin{gathered}
E=\text { const }+\frac{2 \pi\left(\mu_{10}-\mu_{2}\right)^{2} R_{*}^{6}}{3 G_{11} r_{0}^{3}}+ \\
+4 \pi \mu_{2}^{2}\left(\frac{1}{G_{22}}-\frac{1}{G_{11}}\right)\left[\frac{R_{*}^{6}}{6 r_{0}^{3}}+\frac{2 r_{0}^{2}\left(r_{0}^{3}-R_{*}^{3}\right)}{15 R_{2}^{2}}-\frac{R_{*}^{8}}{5 R_{2}^{2} r_{0}^{3}}+\frac{3 R_{*}^{10}}{50 R_{2}^{4} r_{0}^{3}}+\frac{2 r_{0}^{2} R_{*}^{5}}{25 R_{2}^{4}}-\frac{12 r_{0}^{7}}{175 R_{2}^{4}}\right]+E_{s},
\end{gathered}
$$

where $\mu_{2}=m \omega_{0}^{2} R_{2}^{2} / 2, \mu_{10}$ is the chemical potential at $z_{0}=0$ and $R_{*}$ is the droplet radius at $z_{0}=0$. The dependence of the droplet radius on displacement $z_{0}$ is described by Eq. (45).

\section{A. Interface energy}

Here we estimate the interface energy as a function of the droplet displacement $z_{0}$. According to Ref. [19] the interface tension is given by

$$
\sigma=\frac{4}{\sqrt{3}} \sqrt{\left(\xi_{1}^{2}+\xi_{2}^{2}\right)\left[a_{12} / \sqrt{a_{11} a_{22}}-1\right]} P
$$

where $\xi_{i}$ represents the single condensate coherence length $\xi_{i}=\hbar / \sqrt{2 m_{i} G_{i i} n_{i}}$, the pressure $P \approx G_{i i} n_{i}^{2} / 2$ and the condensate densities $n_{i}$ are estimated near the interface. The interface tension $\sigma$ depends on the local density and, hence, changes along the interface. To calculate $\sigma$ we assume that the system is close to the point of phase transition, that is $G_{11} \approx G_{22}=G, R_{1} \approx R_{2}=R, \mu_{1} \approx \mu_{2}=\mu$ and near the interface $n_{1} \approx n_{2}=n$. As a result,

$$
\sigma \approx \frac{2 \hbar \sqrt{G} n^{3 / 2}}{\sqrt{3} \sqrt{m}} \sqrt{a_{12} / \sqrt{a_{11} a_{22}}-1}
$$

and we find for the surface energy

$$
E_{s}=\frac{4 \pi \hbar \mu^{3 / 2} R^{2} r_{0}}{5 \sqrt{3 m} G z_{0}} \sqrt{a_{12} / \sqrt{a_{11} a_{22}}-1}\left[\left(1-\frac{\left(r_{0}-z_{0}\right)^{2}}{R^{2}}\right)^{5 / 2}-\left(1-\frac{\left(r_{0}+z_{0}\right)^{2}}{R^{2}}\right)^{5 / 2}\right] .
$$

\section{B. Energy for small inner droplet}

In the limit $r_{0} \ll R\left(N_{1} \ll N_{2}\right)$ Eq. (45) results in a simple relation between the droplet radius $r_{0}$ and its displacement $z_{0}: r_{0}^{3} \approx \mu_{2} R_{*}^{3} /\left(\mu_{2}-m \omega_{0}^{2} z_{0}^{2} / 2\right)$. Then near the point of phase transition the energy of the system is given by

$$
E \approx \text { const }+N_{1} \mu\left[(1-\kappa) \frac{z_{0}^{2}}{R^{2}}+b\left(1-\frac{z_{0}^{2}}{R^{2}}\right)^{5 / 6}\right]
$$

where

$$
b=\frac{2 \sqrt{6} \xi}{R_{*}} \sqrt{a_{12} / \sqrt{a_{11} a_{22}}-1}=\frac{3 \sigma_{0}}{2 P R_{*}} .
$$

The first term in Eq. (51) corresponds to energy contribution due to difference between $a_{11}$ and $a_{22}$, it increases with $z_{0}$ when $\kappa<1$. The second term is the contribution from the interface energy, it decreases with increasing $z_{0}$ because surface tension drops when the droplet moves in the region with smaller density. In Fig. 10 we plot the energy (51) as a function of displacement of the inner droplet $z_{0}$. The energy is shown for $b=0.3$ and different $\kappa$. Far from the point of phase transition the energy monotonically increases with displacement of the droplet, in this case the first term dominates. When $\kappa$ goes closer to 1 the first term in Eq. (51) becomes small and the interface contribution changes the energy behavior. The energy becomes nonmonotonic function of displacement. The value of $\kappa$ when the final and initial energies are equal is the onset of global instability. However, the system is still locally stable since position 
of the droplet at the center corresponds to a local minima of energy. In this region an energy barrier prevents the droplet to move outside. Further increase of $\kappa$ results in disappearance of the energy barrier and initial configuration becomes both locally and globally unstable.

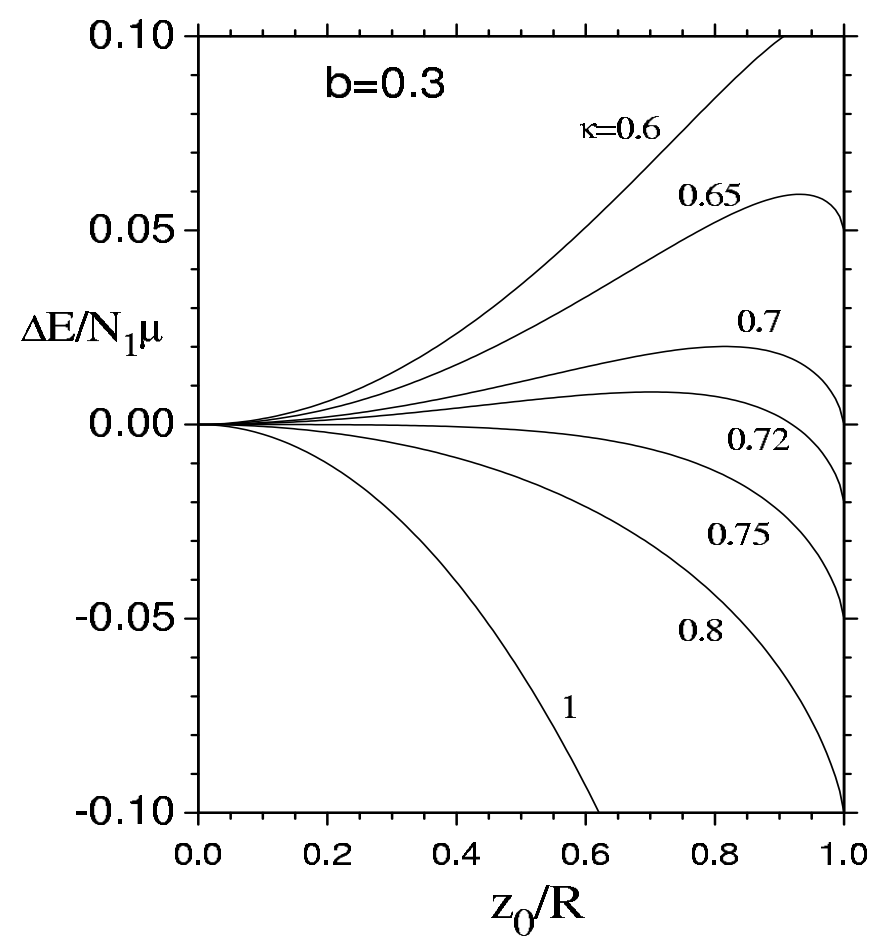

Fig. 10. Energy of the system as a function of displacement of the inner droplet for fixed $b=0.3$ and different $\kappa$.

Near the point of local instability the energy barrier is $\Delta E \approx 18 N_{1} \mu\left(\kappa_{s}-\kappa\right)^{2} / 5 b$, where $\kappa_{s}=1-5 b / 6$. The barrier becomes of the order of $\mu$ when $\kappa_{s}-\kappa \approx \sqrt{5 b / 18 N_{1}}$. If $N_{1} \sim 10^{6}, b \sim 0.1$ we obtain $\kappa_{s}-\kappa \sim 10^{-4}$, while $1-\kappa_{s} \sim 0.1$.

\section{NORMAL MODES FOR DIFFERENT TRAP FREQUENCIES AND PARTICLE MASSES OF THE TWO SPECIES}

In this section we generalize our results to the case $\omega_{1} \neq \omega_{2}, m_{1} \neq m_{2}$ which is useful for future experiments where the trap frequencies and particles can differ for each component. For simplicity we omit the interface tension and consider spherical trapping potentials. The generalized boundary condition for the velocity potential $\Phi$ reads

$$
m_{1} \omega_{1}^{2} R_{*} \frac{\partial \Phi_{1}}{\partial r}-m_{1} \omega^{2} \Phi_{1}=\kappa m_{2} \omega_{2}^{2} R_{*} \frac{\partial \Phi_{2}}{\partial r}-\kappa m_{2} \omega^{2} \Phi_{2},
$$

where $\kappa=\sqrt{G_{11} / G_{22}}=\sqrt{a_{11} m_{2} / a_{22} m_{1}}$. The boundary condition for $\partial \Phi_{1} / \partial r$ remains the same as Eq. (19). Now the phase transition is controlled by a new dimensionless parameter

$$
\tilde{\kappa}=\frac{m_{2} \omega_{2}^{2}}{m_{1} \omega_{1}^{2}} \sqrt{\frac{G_{11}}{G_{22}}}=\frac{m_{2}^{3 / 2} \omega_{2}^{2}}{m_{1}^{3 / 2} \omega_{1}^{2}} \sqrt{\frac{a_{11}}{a_{22}}} .
$$

Instead of (23), the solutions for the inner and outer condensates have the form

$$
\Phi_{1}=C_{1} r^{l} F\left(\alpha_{1}, \beta_{1}, l+3 / 2, \frac{r^{2}}{R_{1}^{2}}\right), \quad \Phi_{2}=C_{2} r^{l} F\left(\alpha_{2}, \beta_{2}, 1,1-\frac{r^{2}}{R_{2}^{2}}\right),
$$

where $R_{i}^{2}=2 \mu_{i} / m_{i} \omega_{i}^{2}(i=1,2)$ and

$$
\alpha_{i}=\frac{1}{2}\left[l+\frac{3}{2}-\sqrt{l^{2}+l+\frac{9}{4}+\frac{2 \omega^{2}}{\omega_{i}^{2}}}\right], \quad \beta_{i}=\frac{1}{2}\left[l+\frac{3}{2}+\sqrt{l^{2}+l+\frac{9}{4}+\frac{2 \omega^{2}}{\omega_{i}^{2}}}\right] .
$$


Using Eq. (53) and the boundary conditions we obtain an equation for the normal mode frequencies

$$
\omega^{2}=\frac{\omega_{1}^{2}(1-\tilde{\kappa})\left[l(l+3 / 2)+\left(l-\omega^{2} / \omega_{1}^{2}\right) \lambda x s_{1}(\omega, x)\right]\left[\left(l-\omega^{2} / \omega_{2}^{2}\right) x s_{2}(\omega, x)-l\right]}{\left[\tilde{\kappa}\left(l-\omega^{2} / \omega_{1}^{2}\right) \lambda x s_{1}(\omega, x) \omega_{1}^{2} / \omega_{2}^{2}+(l+3 / 2)\left(l\left(\tilde{\kappa} \omega_{1}^{2} / \omega_{2}^{2}-1\right)+\left(l-\omega^{2} / \omega_{2}^{2}\right) x s_{2}(\omega, x)\right)\right]},
$$

where

$$
\lambda=\frac{m_{1} \omega_{1}^{2} \mu_{2}}{m_{2} \omega_{2}^{2} \mu_{1}}, \quad x=\frac{R_{*}^{2}}{R_{2}^{2}}, \quad s_{1}(\omega, x)=\frac{F\left(\alpha_{1}+1, \beta_{1}+1, l+5 / 2, \lambda x\right)}{F\left(\alpha_{1}, \beta_{1}, l+3 / 2, \lambda x\right)}, \quad s_{2}(\omega, x)=\frac{F\left(\alpha_{2}+1, \beta_{2}+1,2,1-x\right)}{F\left(\alpha_{2}, \beta_{2}, 1,1-x\right)} .
$$

In terms of $\tilde{\kappa}$ and $\lambda$ the position of the interface is given by

$$
R_{*}=R_{2} \sqrt{\frac{1-\tilde{\kappa} \lambda}{\lambda(1-\tilde{\kappa})}} .
$$

In the region $|1-\tilde{\kappa}| \ll 1$ one can take $\omega=0$ in the right side of Eq. (54) and put $\tilde{\kappa}, \lambda \approx 1$ in the multiple. As a result, we find

$$
\omega^{2} \approx \omega_{1}^{2}(1-\tilde{\kappa}) f(l, x)
$$

where

$$
f(l, x)=\frac{l\left(l+3 / 2+x s_{1}(0, x)\right)\left(x s_{2}(0, x)-1\right)}{\left[x s_{1}(0, x) \omega_{1}^{2} / \omega_{2}^{2}+(l+3 / 2)\left(\omega_{1}^{2} / \omega_{2}^{2}-1+x s_{2}(0, x)\right)\right]}>0 .
$$

Eq. (55) is a generalization of Eq. (25) for the low frequency modes. The point of instability is now determined by the condition $\tilde{\kappa}=1$, or $a_{11} m_{2}^{3} \omega_{2}^{4}=a_{22} m_{1}^{3} \omega_{1}^{4}$. $\tilde{\kappa}$ can be changed not only by changing the relative interaction strengths, but also by changing the ratio of the trapping frequencies. Furthermore it is proportional to a higher (4th) power of the ratio $\omega_{1} / \omega_{2}$ as compared to $a_{11} / a_{22}$ and thus will be very sensitive to the frequency change. Near the instability point $\omega \propto \sqrt{1-\tilde{\kappa}}$ and becomes imaginary when $\tilde{\kappa}>1$.

In summary, we study the normal modes and the stability of the phase separated two component BECs and predict a new symmetric-asymmetric transition in such a system. We show that the normal mode frequencies differ from those for the one component condensate and new branches appear. We found normal modes with low frequencies which are analogous to gravitational waves at the interface between two immiscible fluids. Under the change of the relative interaction strength $a_{11} / a_{22}$ or the ratio $\omega_{1} / \omega_{2}$ the frequencies of those modes go to zero and become imaginary. Imaginary frequencies mean instability and the system undergoes a quantum phase transition into a new stable configuration. Such transition can be observed experimentally by manipulating the interaction strength via Feschbach resonances or by changing the trap frequencies. Interface tension shifts the normal mode frequencies and results in stability of the asymmetric state in some parameter region. Another interesting possibility is to study tunneling by turning on a microwave field which allows direct exchange of the two components and has been done in many JILA experiments with two hyperfine states of ${ }^{87} \mathrm{Rb}$. If we suddenly switch the species, one can study a decay of new unstable configuration or tunneling directly, instead of trying to tune the ratio $a_{11} / a_{22}$.

We are grateful to A. Fetter for valuable remarks. This work was supported by NASA, Grant No. NAG8-1427.

[1] C.J. Myatt, E.A. Burt, R.W. Ghrist, E.A. Cornell, and C.E. Wieman, Phys. Rev. Lett. 78, 586 (1997).

[2] D.S. Hall, M.R. Matthews, J.R. Ensher, C.E. Wieman, and E.A. Cornell, Phys. Rev. Lett. 81, 1539 (1998).

[3] D.M. Stamper-Kurn, M.R. Andrews, A.P. Chikkatur, S. Inouye, H.-J. Miesner, J. Stenger, and W. Ketterle, Phys. Rev. Lett. 80, 2027 (1998).

[4] J. Stenger, S. Inouye, D.M. Stamper-Kurn, H.-J. Miesner, A.P. Chikkatur, and W. Ketterle, Nature (London) 396, 345 (1998).

[5] H.-J. Miesner, D. M. Stamper-Kurn, J. Stenger, S. Inouye, A. P. Chikkatur, and W. Ketterle, Phys. Rev. Lett., 82, 2228, (1999).

[6] D. M. Stamper-Kurn, H.-J. Miesner, A. P. Chikkatur, S. Inouye, J. Stenger, and W. Ketterle, Phys. Rev. Lett., 83, 661, (1999). 
[7] M.R. Matthews, D.S. Hall, D.S. Jin, J.R. Ensher, C.E. Wieman, E.A. Cornell, F. Dalfovo, C. Minniti, and S. Stringari, Phys. Rev. Lett. 81, 243 (1998).

[8] D. S. Hall, M. R. Matthews, C. E. Wieman, and E. A. Cornell, Phys. Rev. Lett., 81, 1543, (1998).

[9] M. R. Matthews, B. P. Anderson, P. C. Haljan, D. S. Hall, C. E. Wieman, and E. A. Cornell, Phys. Rev. Lett., 83, 2498, (1999).

[10] I.M. Khalatnikov, Zh. Éksp. Teor. Fiz. 32, 653 (1957) [Sov. Phys. JETP 5, 542 (1957)].

[11] E.D. Siggia, and A.E. Ruckenstein, Phys. Rev. Lett. 44, 1423 (1980).

[12] T.-L. Ho, and V.B. Shenoy, Phys. Rev. Lett. 77, 3276 (1996).

[13] C. K. Law, H. Pu, N.P. Bigelow, and J.H. Eberly, Phys. Rev. Lett. 79, 3105 (1997).

[14] B.D. Esry, C.H. Greene, J.P. Burke, and J.L. Bohn, Phys. Rev. Lett. 78, 3594 (1997).

[15] E.V. Goldstein, and P. Meystre, Phys. Rev. A 55, 2935 (1997).

[16] P. Öhberg, and S. Stenholm, Phys. Rev. A. 57, 1272 (1998).

[17] H. Pu, and N.P. Bigelow, Phys. Rev. Lett. 80, 1130 (1998).

[18] P. Ao, and S. T. Chui, Phys. Rev. A 58, 4836 (1998).

[19] E. Timmermans, Phys. Rev. Lett. 81, 5718 (1998).

[20] S. T. Chui and P. Ao, Phys. Rev. A, 59, 1473, (1999).

[21] S. T. Chui, P. Ao, and B. Tanatar, J. Phys. Soc. Jpn., 15, 142, 1999.

[22] T. Busch, J.I. Cirac, V.M. Pérez-García, and P. Zoller, Phys. Rev. A 56, 2978 (1997).

[23] R. Graham, and D. Walls, Phys. Rev. A 57, 484 (1998).

[24] B.D. Esry, and C.H. Greene, Phys. Rev. A 57, 1265 (1998).

[25] D. Gordon, and C.M. Savage, Phys. Rev. A 58, 1440 (1998).

[26] I.E. Mazets, Phys. Rev. A 65, 033618 (2002).

[27] H. Pu, and N.P. Bigelow, Phys. Rev. Lett. 80, 1134 (1998).

[28] J.P. Burke, Jr., J.L. Bohn, B.D. Esry, and C.H. Greene, Phys. Rev. A 55, R2511 (1997).

[29] S.T. Chui, V.N. Ryzhov, and E.E. Tareyeva, Pis'm. Zh. Éksp. Teor. Fiz. 75, 279 (2002) [JETP Letters 75, 233 (2002)].

[30] A.L. Fetter, Phys. Rev. A 53, 4245 (1996).

[31] S. Stringari, Phys. Rev. Lett. 77, 2360 (1996).

[32] L. D. Landau, and E. M. Lifshits, Hydrodynamics, Moscow, Nauka, (1988), Sec. 12.

[33] P. Ao and S. T. Chui, J. Phys. B: At. Mol. Opt. Phys. 33, 535 (2000).

[34] L.D. Landau, and E.M. Lifshits, Hydrodynamics, Moscow, Nauka, (1988), Sec. 62. 